\title{
Retrospective Comparison of Midterm Clinical and Angiographic Outcomes after the Implantation of Paclitaxel- and Sirolimus-Eluting Stents for de novo Coronary Complex Lesions in Nonrandomized Japanese Patients
}

Tetsuya Ishikawa, Yosuke Nakano and Makoto Mutoh

\begin{abstract}
Objective We retrospectively analyzed the midterm clinical and angiographic outcomes after the implantation of paclitaxel- (PES, TAXUS Express) and sirolimus- (SES, Cypher Bx Velocity) eluting stents in Japanese patients with complex coronary lesions.

Methods From August 2004 to May 2009, 1,335 nonrandomized de novo native complex coronary lesions treated with either a PES (357 cases) or SES were included. The inclusion criteria for patients with complex lesions were those with diabetes, those undergoing hemodialysis, and those with a low ejection fraction, as well as subjects who had lesions with severe calcification, ostiums of the right coronary and left circumflex arteries, and who underwent the side-branch bifurcation 2-stent technique. The subjects were evaluated for consistent predictors of cardiac events, binary restenosis and target lesion vascularization of the SES. The composite primary endpoint percentage (900-day cardiac death, nonfatal recurrent myocardial infarction, and definite stent thrombosis) after PES placement was $0.6 \%$, which was not significantly different from that after SES placement $(0.12 \% ; \mathrm{p}=0.290)$. The incidence of the secondary endpoint (binary restenosis; stenosis > $50 \%$ of the diameter at the secondary angiographic examination performed within 550 days after the procedure) after PES placement (15.0\%) was also not significantly different than that after SES placement (13.3\%; $\mathrm{p}=0.498$ ). There was no relationship between PES placement and binary restenosis upon angiographic followup of 989 lesions (odds ratio of 1.14; 95\% confidence interval, 0.73-1.77; $\mathrm{p}=0.57$ ).

Conclusion For de novo native complex coronary stenosis, the midterm safety and efficacy of PES placement was statistically equivalent to that of SES placement in a clinical setting in Japan.
\end{abstract}

Key words: paclitaxel-eluting stent, sirolimus-eluting stent, angioplasty, restenosis, stent thrombosis, followup result

(Intern Med 51: 2695-2701, 2012)

(DOI: 10.2169/internalmedicine.51.6704)

\section{Introduction}

The paclitaxel-eluting stent (PES) (TAXUS Express; Boston Scientific, Natick, MA, USA) was the second coronary drug-eluting stent (DES) to be approved after the sirolimuseluting stent (SES) (Cypher Bx Velocity; Cordis Corp., Miami, FL, USA) in Japan. Previous studies have compared the outcomes of PES to those of SES in Japanese patients $(1,2)$. However, no studies in Japan have compared the safety and efficacy of PES versus SES for the treatment of de novo complex lesions in native coronary arteries, which are widely treated with DES.

SES, the first-approved DES, markedly reduces the incidence of binary in-stent restenosis (binary restenosis) and target lesion revascularization (TLR) compared to bare-metal 
stents, without increasing the mid-term adverse cardiac events, including stent thrombosis (ST), in Japan (3). However, following the widespread application of percutaneous coronary intervention (PCI), novel problems have been raised about using DES for complex lesions in patients considered to have predictors of severe cardiac events, binary restenosis, and TLR of SES, such as diabetes, hemodialysis, and a low ejection fraction (EF), as well as those with lesions with severe calcification, ostiums of the right coronary and left circumflex arteries, and who have undergone the side-branch bifurcation 2-stent technique (3-5). Therefore, in order to improve the rate of complete coronary revascularization by combining the advantages of DESs, it was necessary to examine the impact of the novel DES approved after SES, PES, on those complex lesions.

Therefore, the present study examined the midterm clinical and angiographic outcomes of PES versus SES in Japanese patients with complex coronary lesions, comprising those with consistent predictors of severe cardiac events, binary restenosis, and TLR (3-5). We retrospectively analyzed the 900-day clinical and 550-day angiographic outcomes after PES and SES implantations in patients with complex coronary lesions.

\section{Materials and Methods}

\section{Study design and population}

The present study was a nonrandomized, single-center (Saitama Cardiovascular and Respiratory Center), lesionbased, retrospective study conducted in December 2011. The inclusion criteria were patients with de novo stenosis in native coronary arteries that were successfully and exclusively treated by elective PES or SES and who had not undergone a prior coronary artery bypass graft $(\mathrm{CABG})$. Treatment was considered successful based on the absence of periprocedural complications [i.e., death, Q-wave myocardial infarction (MI), and emergency CABG]. Furthermore, patients were enrolled when post-procedural antegrade coronary flow determined thrombolysis in myocardial infarction (TIMI) grade 3 flow with acceptable stent expansion, as determined by angiography and IVUS. Complex lesions were defined based on previous data identifying the predictors of binary restenosis as TLR, a severe cardiac event after SES treatment (3-5), patients with diabetes, hemodialysis, and a low $\mathrm{EF}$, and lesions with severe calcification, ostiums of the right coronary and left circumflex arteries, and previous use of the side-branch bifurcation 2-stent technique. In addition, patients who had lesions with a post-procedural lumen diameter $>1.35 \mathrm{~mm}$, stenosis of $<40 \%$ of the diameter, and a reference diameter $\leq 3.75 \mathrm{~mm}$ were included. The cases who underwent PCI supported by intra-aortic balloon pumping were excluded.

From August 2004 (the time of SES approval in Japan) to May 2007 [the time of PES (Express) approval in Japan], SES were successfully placed in 767 complex lesions. From
May 2007 to February 2009 [before TAXUS (Liberte) was approved in Japan], PES were successfully placed in 357 lesions, and SES were placed in 184 lesions. From February 2009 to August 2009, SES were placed in 27 lesions. Together, 1,335 consecutive, nonrandomized, complex lesions (PES group, $\mathrm{n}=357$; SES group, $\mathrm{n}=978$ ) in 840 patients were treated and eligible for the study, and were therefore included in the analyses.

\section{Procedures for stenting and antiplatelet therapy}

All patients were informed of the necessity of PCI and stenting, and informed consent was obtained. The device used to successfully treat the lesion by stenting was subject to the doctor's discretion. During the procedure, heparin (5,000-8,000 IU) was administered. The stents were implanted by visual angiographic estimation to cover the entire baseline lesion under the guidance of intravascular ultrasonography (IVUS-guide) (Table 1). When further stent dilation was needed, high-pressure ballooning with a noncompliant balloon (6) was typically performed. Rotational atherectomy with a rotablator was used (i) when it was impossible to insert the devices (a small-sized balloon and/or IVUS device) and (ii) when the balloon used for pre-dilation of the target lesion was indented (7).

Periprocedural antiplatelet therapy was conducted as previously reported $(3,4)$. When elective PCI was planned, aspirin (81-100 mg) and ticlopidine (200 mg) were orally administered approximately 10 days before the index procedure. After the procedure, ticlopidine (200 mg/day) was prescribed for a minimum of 12 months, although these prescriptions were not prospectively randomized. Cilostazol (200-300 mg/day) or clopidogrel (75 mg/day) was administered at the doctor's discretion if the patient had any adverse reactions to ticlopidine. From November 2007 to March 2008, clopidogrel was the first-line thienopyridine agent.

\section{Follow-up angiogram and quantitative coronary ar- tery (QCA) evaluation}

The angiographic outcomes at the follow-up angiogram (fu CAG) through December 2011 were included in the analyses. The fu CAG was planned approximately 8-18 months (within 550 days) after the procedure. The rate of fu CAG was $70.3 \%$ (257 among 357 cases) in the PES group and $76.6 \%$ in the SES (749 among 978 cases) group (no significant difference; $\mathrm{p}=0.084$ ). The QCA parameters were measured using a TCS cardiovascular network system (CAAS-2 and CAAS-5 system, Netherlands), as described previously $(3,4,8)$. Values were obtained at three time points: before PCI (pre-procedural), immediately after successful PCI (post-procedural), and during the chronic phase (follow-up). The measurements included the minimal lumen diameter (MLD), \% diameter stenosis (\% DS), and reference diameter (RD). In cases showing occlusions at the preprocedure and follow-up stages, the MLD was considered 0, and the \%DS was considered 100. Additionally, we calculated the acute gain (post-procedural MLD minus pre- 
Table 1. Lesion-based Baseline Characteristics and Clinical Outcomes in 1,335 de novo Native Complex Coronary Stenosis Lesions that Underwent PES or SES Placement

\begin{tabular}{|c|c|c|c|}
\hline & $\begin{array}{c}\text { PES } \\
357\end{array}$ & $\begin{array}{c}\text { SES } \\
978\end{array}$ & $p$ value \\
\hline Age (yr) & $67.8 \pm 9.4$ & $67.6 \pm 9.1$ & 0.729 \\
\hline Male gender (\%) & 75.1 & 76.5 & 0.592 \\
\hline Diabetes (\%) & 77.6 & 79.0 & 0.568 \\
\hline OMI (\%) & 48.7 & 44.5 & 0.167 \\
\hline Hemodialysis (\%) & 3.1 & 3.2 & 0.934 \\
\hline Low EF (\%) & 4.8 & 6.0 & 0.463 \\
\hline LAD (\%) & 37.0 & 39.3 & 0.447 \\
\hline RCA (\%) & 32.8 & 26.9 & $<0.05$ \\
\hline Calcification (\%) & 21.6 & 20.9 & 0.778 \\
\hline RCA ostium (\%) & 4.5 & 3.0 & 0.174 \\
\hline LCX ostium (\%) & 8.4 & 6.7 & 0.300 \\
\hline Bifurcation (\%) & 43.4 & 52.5 & $<0.01$ \\
\hline СTO $(\%)$ & 8.4 & 9.5 & 0.536 \\
\hline Side branch stenting (\%) & 11.5 & 12.9 & 0.494 \\
\hline IVUS (\%) & 96.6 & 96.7 & 0.935 \\
\hline Rotablator (\%) & 11.5 & 15.1 & 0.091 \\
\hline Number of stents & $1.46 \pm 0.76$ & $1.45 \pm 0.67$ & 0.826 \\
\hline Diameter of balloon $(\mathrm{mm})$ & $3.09 \pm 0.41$ & $3.08 \pm 0.45$ & 0.701 \\
\hline Length of stent (mm) & $35.6 \pm 21.5$ & $35.5 \pm 19.4$ & 0.938 \\
\hline Pressure (atm) & $18.1 \pm 3.03$ & $19.9 \pm 3.13$ & $<0.001$ \\
\hline Pre-procedural MLD (mm) & $0.94 \pm 0.49$ & $0.93 \pm 0.54$ & 0.631 \\
\hline Pre-procedural \%DS & $64.6 \pm 17.9$ & $64.9 \pm 18.5$ & 0.787 \\
\hline Post-procedural MLD (mm) & $2.52 \pm 0.42$ & $2.48 \pm 0.39$ & 0.117 \\
\hline Acute gain $(\mathrm{mm})$ & $1.58 \pm 0.60$ & $1.56 \pm 0.52$ & 0.577 \\
\hline Post-procedural \%DS & $11.4 \pm 8.5$ & $12.3 \pm 9.0$ & $<0.01$ \\
\hline Post-procedural RD (mm) & $2.86 \pm 0.51$ & $2.85 \pm 0.46$ & 0.750 \\
\hline Clinical observational interval (day) & $873 \pm 133$ & $879 \pm 112$ & 0.447 \\
\hline Primary endpoint $(\mathrm{n}, \%)$ & $2,0.6$ & $12,0.12$ & 0.290 \\
\hline Cardiac death $(\mathrm{n}, \%)$ & $2,0.6$ & $12,0.12$ & 0.290 \\
\hline Recurrent non-fatal MI (n, \%) & 0 & 0 & \\
\hline Definite stent thrombosis ( $\mathrm{n}, \%)$ & 0 & $1,0.10$ & 0.546 \\
\hline
\end{tabular}

26 baseline variables related to patients, lesions, and procedures and 5 variables related to the primary endpoint are shown. The definitions of the variables are as follows: age (age at stenting); male gender; diabetes (patients with diabetes mellitus); low EF (ejection fraction of left ventricle less than 40 as evaluated by ultrasonography or left ventriculogram); location of the culprit lesion in the left anterior descending artery (LAD) and right coronary artery (RCA); calcification (moderate to severe calcified lesions estimated using an angiogram and IVUS); ostial lesions of left circimflex artery (LCX ostium) and RCA (RCA ostium); bifurcation (bifurcative lesions requiring treatment of the side branch); CTO (chronic total occlusion more than 3 months); side-branch stenting (side branch of bifurcation 2-stent technique); IVUS (availability of IVUS during PCI); number of stents (number of implanted stents per lesion); diameter of balloon (maximum diameter of the balloon used to dilate the stent); length of stent (length of the stented segment calculated by adding the lengths of each stent regardless of overlap); pressure (maximum pressure at the maximum diameter of the inflated balloon); clinical observational interval (duration in days monitored after the index procedure). The lesion location, calcification, bifurcation, ostial lesion, and CTO variables were defined according to the American College of Cardiology and/or American Heart Association classification of lesions. Before PCI (pre-procedural) and immediately after successful PCI (post-procedural), the minimal lumen diameter (MLD), \% diameter stenosis (DS), and reference diameter (RD) were assessed. Additionally, we calculated acute gain (post-procedural MLD minus pre-procedural MLD). The definition of the primary endpoint is provided in the text.

procedural MLD) and late luminal loss (post-procedural MLD minus MLD at the chronic phase). Binary restenosis
Table 2. Baseline Characteristics of Lesions with or without Primary Endpoints (event) after Stenting with PES or SES

\begin{tabular}{|c|c|c|c|}
\hline & $\begin{array}{c}\text { Event } \\
14\end{array}$ & $\begin{array}{c}\text { No event } \\
1,321\end{array}$ & $\mathrm{p}$ value \\
\hline Age (yr) & $73.1 \pm 7.1$ & $67.6 \pm 9.2$ & $<0.01$ \\
\hline Male gender (\%) & 71.4 & 76.2 & 0.680 \\
\hline Diabetes (\%) & 78.6 & 78.7 & 0.994 \\
\hline OMI (\%) & 50.0 & 45.6 & 0.741 \\
\hline Hemodialysis (\%) & 14.3 & 3.0 & $<0.05$ \\
\hline Low EF (\%) & 35.7 & 5.4 & $<0.001$ \\
\hline LAD (\%) & 28.6 & 38.8 & 0.436 \\
\hline RCA (\%) & 42.9 & 28.3 & 0.230 \\
\hline Calcification (\%) & 35.7 & 20.9 & 0.176 \\
\hline RCA ostium (\%) & 7.1 & 3.3 & 0.432 \\
\hline LCX ostium (\%) & 7.1 & 7.2 & 0.994 \\
\hline Bifurcation (\%) & 21.4 & 50.3 & $<0.05$ \\
\hline Сто $(\%)$ & 7.1 & 9.2 & 0.788 \\
\hline Side branch stenting (\%) & 0 & 12.6 & 0.155 \\
\hline IVUS (\%) & 78.6 & 96.9 & $<0.001$ \\
\hline Rotablator (\%) & 7.1 & 14.2 & 0.449 \\
\hline PES (\%) & 14.3 & 26.9 & 0.290 \\
\hline Number of stents & $1.36 \pm 0.63$ & $1.45 \pm 0.70$ & 0.595 \\
\hline Diameter of balloon $(\mathrm{mm})$ & $3.20 \pm 0.34$ & $3.09 \pm 0.44$ & 0.230 \\
\hline Length of stent (mm) & $30.8 \pm 17.5$ & $35.6 \pm 20.0$ & 0.308 \\
\hline Pressure (atm) & $19.1 \pm 3.66$ & $19.4 \pm 3.20$ & 0.760 \\
\hline Pre-procedural MLD (mm) & $1.02 \pm 0.63$ & $0.93 \pm 0.52$ & 0.594 \\
\hline Pre-procedural \%DS & $64.9 \pm 18.6$ & $64.8 \pm 18.3$ & 0.984 \\
\hline Post-procedural MLD (mm) & $2.66 \pm 0.40$ & $2.49 \pm 0.40$ & 0.114 \\
\hline Acute gain $(\mathrm{mm})$ & $1.65 \pm 0.60$ & $1.56 \pm 0.54$ & 0.576 \\
\hline Post-procedural \%DS & $13.3 \pm 8.0$ & $12.1 \pm 8.9$ & 0.577 \\
\hline Post-procedural RD (mm) & $3.08 \pm 0.39$ & $2.85 \pm 0.47$ & $<0.05$ \\
\hline Clinical observational interval (day) & $249 \pm 198$ & $884 \pm 97$ & $<0.001$ \\
\hline Primary endpoint (n, \%) & 100 & 0 & \\
\hline
\end{tabular}

Variables related to patients, clinical conditions, lesions, and procedure are shown for the event and no-event groups. Abbreviations are defined in Table 1.

was defined as a $\%$ DS $>50$ at the fu CAG. Severe stenosis (\% DS $\geq 70$ at follow-up), typically treated as clinical TLR, was also assessed. The incidence of TLR after the fu CAG was assessed mainly by visual estimation of angiographic outcomes.

\section{Endpoints and baseline variables of the enrolled pa- tients}

The safety (primary) endpoint of the clinical outcome was the cardiac-event composite comprising target vessel-related death without a definite non-cardiac cause (cardiac death), nonfatal re-MI, and definite (early, late, and very late) ST, as defined by the ARC (9), within 900 days after the index procedure. The efficacy (secondary) endpoint of the angiographic outcome was defined as the incidence of binary restenosis within 550 days after the index procedure.

\section{Statistical analyses}

The baseline characteristics are expressed as the means \pm standard deviation (SD). The PES and SES group variables (both at baseline and at angiographic follow-up) were compared using unpaired $t$-tests for continuous values and $\chi^{2}$ tests for categorical values. The present study compared the safety and efficacy between SES and PES. Therefore, the 
Table 3. Predictors of the Primary Endpoint

\begin{tabular}{cccc}
\hline & Hazard ratio & $95 \% \mathrm{Cl}$ & $\mathrm{p}$ value \\
\hline Low EF & 7.56 & $2.46-23.2$ & $<0.001$ \\
IVUS & 0.17 & $0.04-0.65$ & $<0.05$ \\
Hemodialysis & 4.80 & $1.00-23.0$ & $<0.05$ \\
Age & 1.08 & $1.01-1.17$ & $<0.05$ \\
\hline Bifurcation & 0.35 & $0.10-1.30$ & 0.118 \\
Post-procedural RD & 2.38 & $0.76-7.51$ & 0.139 \\
PES & 0.43 & $0.09-1.94$ & 0.272 \\
\hline
\end{tabular}

Predictors of the primary endpoint analyzed using the Cox proportional hazard model are shown. The first 4 variables were significant predictors according to the multivariate analysis, and the following 3 variables were predictors according to the univariate analysis. Abbreviations are defined in Table 1 and text.

following steps were used to create a multivariate model: (a) clinical and angiographic variables were compared between endpoint-positive (event and binary restenosis) and endpointnegative (no event and no binary restenosis) groups (Table 2,5$)$. The $\mathrm{p}$ values for each comparison were calculated using either the $\chi^{2}$ tests (Chi and Fisher's test) or $t$-tests (univariate analysis). (b) A multivariate analysis for predicting adverse safety and efficacy endpoints was performed. The stent type (PES) was included as an independent variable, and other variables found to be significant according to the univariate analysis were added (Table 2, 5). The Cox proportional hazard model was used by including seven variables (age, low EF, hemodialysis, IVUS, bifurcation, post-procedural RD, and PES) in order to analyze the predictors of the primary endpoint (Table 3 ). The predictors of binary restenosis were analyzed using a logistic regression analysis by including 12 variables (hemodialysis, LAD, RCA ostium, LCx ostium, CTO, side-branch stenting, PES, number of stents, length of stent(s), pre-procedural MLD, pre-procedural \%DS, and acute gain) (Table 6). A p value $<0.05$ was considered to be statistically significant. The Stata version 8 software program for Windows (StataCorp, College Station, TX, USA) was used for the statistical analyses.

\section{Results}

\section{Baseline characteristics and primary endpoints}

Table 1 shows the baseline characteristics and primary endpoints of the PES $(n=357)$ and SES $(n=978)$ groups. Among the patients, lesions, and procedural characteristics, the percentage of RCA (32.8\%), bifurcation (43.4\%), and mean pressure $(18.1 \pm 3.03 \mathrm{~atm})$ in the PES group were significantly different than those in the SES group (26.9\%, $52.5 \%$, and $19.9 \pm 3.13 \mathrm{~atm}$, respectively) $(\mathrm{p}<0.05,<0.01$, and $<0.001$, respectively).

The lesion-based incidence of the primary endpoint in the PES group (0.6\%; mean follow-up of $873 \pm 133$ days) was not significantly different from that in the SES group (0.12\%, $\mathrm{p}=0.447$; mean follow-up of $879 \pm 112$ days).

\section{Baseline characteristics of lesions with and without a primary endpoint}

Table 2 shows the baseline characteristics of lesions with primary endpoints (event group) $(n=14)$ and without primary endpoints (no-event group) $(\mathrm{n}=1,321)$. Among the variables, the percentage of patients on hemodialysis $(14.3 \%)$, with a low EF (35.7\%), bifurcation (21.4\%), and IVUS (78.6\%) as well as the age $(73.1 \pm 7.1 \mathrm{yr})$, post-procedural RD (3.08 \pm $0.39 \mathrm{~mm})$, and clinical observational interval $(249 \pm 198$ days) in the event group were significantly different from those in the no-event group $(3.0 \%, 5.4 \%, 50.3 \%, 96.9 \%$, $67.6 \pm 9.2 \mathrm{yr}, 2.85 \pm 0.47$, and $880 \pm 112$ day, respectively) $(\mathrm{p}<$ $0.05,<0.001,<0.05,<0.001,<0.01,<0.05$, and $<0.001$, respectively).

\section{Predictors of the primary endpoint}

Table 3 shows the results of the Cox proportional hazard model for identifying predictors of the primary endpoint. Among the 1,335 lesions, a low EF [hazard ratio (HR), 7.56; 95\% confidence interval (CI), 2.46-23.2; p<0.001), IVUS (HR, 0.17; 95\% CI, 0.04-0.65; p<0.05), hemodialysis (HR, 4.80; 95\% CI, 1.00-23.0; $\mathrm{p}<0.05)$, and age (HR, 1.08; 95\% CI, 1.01-1.17; $\mathrm{p}<0.05)$ were significant predictors of the primary endpoint. Bifurcation, post-procedural RD, and PES were not significantly related to the primary endpoint based on the multivariate analysis.

\section{Effects of baseline characteristics on angiographic follow-up lesions and angiographic outcomes}

The baseline characteristics of 989 lesions with angiographic follow-up and the angiographic outcomes with secondary endpoints in the PES group $(n=246)$ and SES group $(n=743)$ are shown in Table 4 . The incidence of bifurcation $(45.6 \%)$ in the PES group was significantly lower than in the SES group $(54.4 \% ; \mathrm{p}<0.05)$. The mean pressure $(18.0 \pm$ $3.06 \mathrm{~atm})$, follow-up MLD $(2.01 \pm 0.67 \mathrm{~mm})$, and late luminal loss $(0.48 \pm 0.68 \mathrm{~mm})$ in the PES group were significantly different from those in the SES group (19.8 \pm 3.12 atm, $2.17 \pm 0.72 \mathrm{~mm}$, and $0.32 \pm 0.68 \mathrm{~mm}$, respectively) $(\mathrm{p}<$ $0.001,<0.01$, and $<0.001$, respectively).

The incidence of binary restenosis in the PES group $(15.0 \%)$ did not significantly differ from that in the SES group $(13.3 \% ; \mathrm{p}=0.498)$. The incidence of severe restenosis in the PES group $(4.1 \%)$ was also not significantly different from that in the SES group (5.0\%; $\mathrm{p}=0.559)$, nor was the incidence of TLR in the PES group (12.6\%) significantly different from that in the SES group (12.0\%; $\mathrm{p}=0.795)$.

\section{Baseline characteristics of lesions with and without binary restenosis}

Table 5 shows the baseline characteristics of lesions with the secondary endpoint (binary restenosis group) $(n=136)$ and without the secondary endpoint (no binary restenosis 
Table 4. Baseline Characteristics and Serial Quantitative Coronary Angiography Findings after Follow-up Secondary Angiography in 989 Lesions Stented with PES or SES

\begin{tabular}{|c|c|c|c|}
\hline & PES & SES & \\
\hline & 246 & 743 & $p$ value \\
\hline Age (yr) & $66.5 \pm 9.5$ & $66.5 \pm 8.8$ & 1.000 \\
\hline Male gender (\%) & 78.9 & 78.5 & 0.896 \\
\hline Diabetes (\%) & 80.9 & 78.6 & 0.443 \\
\hline OMI (\%) & 50.8 & 45.5 & 0.147 \\
\hline Hemodialysis (\%) & 1.6 & 3.4 & 0.188 \\
\hline Low EF (\%) & 4.1 & 4.7 & 0.674 \\
\hline LAD (\%) & 37.4 & 39.3 & 0.596 \\
\hline RCA (\%) & 31.3 & 26.5 & 0.146 \\
\hline Calcification (\%) & 20.7 & 19.7 & 0.713 \\
\hline RCA ostium (\%) & 3.3 & 3.0 & 0.818 \\
\hline LCX ostium (\%) & 8.1 & 7.1 & 0.142 \\
\hline Bifurcation (\%) & 45.6 & 54.4 & $<0.05$ \\
\hline Side-branch stenting (\%) & 12.6 & 12.8 & 0.835 \\
\hline сто (\%) & 9.3 & 9.6 & 0.924 \\
\hline IVUS (\%) & 95.5 & 96.8 & 0.361 \\
\hline Rotablator (\%) & 11.8 & 11.3 & 0.185 \\
\hline Number of stents & $1.44 \pm 0.73$ & $1.44 \pm 0.67$ & 1.000 \\
\hline Diameter of balloon ( $\mathrm{mm}$ ) & $3.05 \pm 0.40$ & $3.09 \pm 0.46$ & 0.191 \\
\hline Length of stent (mm) & $34.9 \pm 20.8$ & $35.5 \pm 19.3$ & 0.690 \\
\hline Pressure (atm) & $18.0 \pm 3.06$ & $19.8 \pm 3.12$ & $<0.001$ \\
\hline Pre-procedural MLD (mm) & $0.94 \pm 0.51$ & $0.93 \pm 0.54$ & 0.793 \\
\hline Pre-procedural \% DS & $64.9 \pm 17.9$ & $64.9 \pm 18.4$ & 1.000 \\
\hline Post-procedural MLD (mm) & $2.49 \pm 0.43$ & $2.48 \pm 0.39$ & 0.746 \\
\hline Acute gain $(\mathrm{mm})$ & $1.56 \pm 0.64$ & $1.55 \pm 0.52$ & 0.824 \\
\hline Post-procedural \% DS & $11.3 \pm 8.64$ & $12.5 \pm 9.14$ & 0.063 \\
\hline Post-procedural RD (mm) & $2.83 \pm 0.51$ & $2.85 \pm 0.45$ & 0.583 \\
\hline Follow-up MLD (mm) & $2.01 \pm 0.67$ & $2.17 \pm 0.72$ & $<0.01$ \\
\hline Late luminal loss $(\mathrm{mm})$ & $0.48 \pm 0.68$ & $0.32 \pm 0.68$ & $<0.01$ \\
\hline Follow-up \% DS & $28.9 \pm 19.3$ & $27.6 \pm 19.6$ & 0.362 \\
\hline Binary restenosis $(\%)$ & 15.0 & 13.3 & 0.498 \\
\hline Severe restenosis $(\%)$ & 4.1 & 5.0 & 0.559 \\
\hline Target lesion revascularization (\%) & 12.6 & 12.0 & 0.795 \\
\hline $\begin{array}{l}\text { Interval for angiographic follow-up } \\
\text { (day) }\end{array}$ & $379 \pm 150$ & $365 \pm 156$ & 0.209 \\
\hline
\end{tabular}

29 baseline variables related to patients, lesions, and procedures and 4 variables related to the secondary endpoint are shown. The definitions of the variables are described in Table 1 and as follows: interval for angiographic follow-up (duration in days after the index procedure). Before PCI (pre-procedural), immediately after successful PCI (post-procedural), and at the chronic phase (follow-up), the minimal lumen diameter (MLD), \% diameter stenosis (DS), and reference diameter (RD) were assessed. Lesion length (LL) was obtained at 2 points (preprocedure and at follow-up). Additionally, we calculated acute gain and late luminal loss (post-procedural MLD minus MLD at chronic phase). Binary in-stent restenosis (binary restenosis) was defined as \% DS $>50$ at fu CAG. Severe stenosis (\% DS $\geq 70$ at follow-up), typically treated as clinical target lesion revascularization (TLR), was also compared. TLR was evaluated mainly by visual estimation of angiographic outcomes.

group) ( $\mathrm{n}=853)$. The percentages of patients receiving hemodialysis (8.8\%), with LAD (30.9\%), RCA ostium (5.9\%), LCX ostium (19.1\%), CTO (16.2\%), side-branch stenting $(23.5 \%)$, and severe restenosis $(34.6 \%)$ in the binary restenosis group were significantly different from those in the no binary restenosis group $(2.0 \%, 40.1 \%, 2.6 \%, 5.5 \%$, $8.3 \%, 11.1 \%$, and $0 \%$, respectively) $(\mathrm{p}<0.001,<0.05,<0.05$, $<0.05,<0.001$, and $<0.001$, respectively). The mean number of stents $(1.74 \pm 0.84)$, stent length $(43.7 \pm 23.8)$, preprocedural MLD $(0.82 \pm 0.59 \mathrm{~mm})$, pre-procedural \%DS $(69.4 \pm 20.8)$, acute gain $(1.69 \pm 0.62 \mathrm{~mm})$, follow-up MLD
Table 5. Baseline Characteristics and Serial Quantitative Coronary Angiography Findings after Follow-up Secondary Angiography of Lesions with or without Binary Restenosis after Stenting with PES or SES

\begin{tabular}{|c|c|c|c|}
\hline & $\begin{array}{c}\text { Binary } \\
\text { restenosis } \\
136\end{array}$ & $\begin{array}{c}\text { No binary } \\
\text { restenosis } \\
853\end{array}$ & $\mathrm{p}$ value \\
\hline Age (yr) & $65.9 \pm 9.4$ & $66.6 \pm 8.9$ & 0.417 \\
\hline Male gender (\%) & 76.5 & 78.9 & 0.522 \\
\hline Diabetes (\%) & 80.1 & 79.0 & 0.763 \\
\hline OMI (\%) & 50.0 & 46.3 & 0.423 \\
\hline Hemodialysis (\%) & 8.8 & 2.0 & $<0.001$ \\
\hline Low EF (\%) & 6.6 & 4.2 & 0.213 \\
\hline LAD (\%) & 30.9 & 40.1 & $<0.05$ \\
\hline RCA (\%) & 34.6 & 26.6 & 0.054 \\
\hline Calcification (\%) & 25.0 & 19.1 & 0.110 \\
\hline RCA ostium (\%) & 5.9 & 2.6 & $<0.05$ \\
\hline LCX ostium (\%) & 19.1 & 5.5 & $<0.001$ \\
\hline Bifurcation (\%) & 55.9 & 51.0 & 0.290 \\
\hline СтО (\%) & 16.2 & 8.4 & $<0.01$ \\
\hline Side branch stenting (\%) & 23.5 & 11.0 & $<0.001$ \\
\hline IVUS (\%) & 97.8 & 96.2 & 0.365 \\
\hline Rotablator (\%) & 18.4 & 13.7 & 0.150 \\
\hline PES (\%) & 27.2 & 24.5 & 0.498 \\
\hline Number of stents & $1.74 \pm 0.84$ & $1.40 \pm 0.64$ & $<0.001$ \\
\hline Diameter of balloon (mm) & $3.09 \pm 0.37$ & $3.08 \pm 0.46$ & 0.610 \\
\hline Length of stent (mm) & $43.7 \pm 23.8$ & $34.0 \pm 18.6$ & $<0.001$ \\
\hline Pressure (atm) & $19.4 \pm 3.6$ & $19.4 \pm 3.1$ & 0.754 \\
\hline Pre-procedural MLD (mm) & $0.82 \pm 0.59$ & $0.95 \pm 0.52$ & $<0.05$ \\
\hline $\begin{array}{l}\text { Pre-procedural \% DS } \\
\text { Post-procedural MLD }\end{array}$ & $69.4 \pm 20.8$ & $64.2 \pm 17.8$ & $<0.01$ \\
\hline$(\mathrm{mm})$ & $2.50 \pm 0.37$ & $2.48 \pm 0.41$ & 0.564 \\
\hline Acute gain $(\mathrm{mm})$ & $1.69 \pm 0.62$ & $1.53 \pm 0.54$ & $<0.01$ \\
\hline Post-procedural \% DS & $12.5 \pm 9.4$ & $12.1 \pm 9.0$ & 0.643 \\
\hline Post-procedural RD (mm) & $2.88 \pm 0.44$ & $2.84 \pm 0.47$ & 0.329 \\
\hline Follow-up MLD (mm) & $0.95 \pm 0.48$ & $2.32 \pm 0.54$ & $<0.001$ \\
\hline Late luminal loss (mm) & $1.55 \pm 0.54$ & $0.17 \pm 0.49$ & $<0.001$ \\
\hline Follow-up \% DS & $68.1 \pm 14.8$ & $21.5 \pm 10.4$ & $<0.001$ \\
\hline Binary restenosis (\%) & 100.0 & 0 & $<0.001$ \\
\hline Follow-up $\%$ DS $\geq 70$ (\%) & 34.6 & 0 & $<0.001$ \\
\hline $\operatorname{TLR}(\%)$ & 82.4 & 0.9 & $<0.001$ \\
\hline $\begin{array}{l}\text { Interval for angiographic } \\
\text { follow-up (day) }\end{array}$ & $352 \pm 183$ & $371 \pm 149$ & 0.250 \\
\hline
\end{tabular}

Variables related to patients, clinical conditions, lesions, and procedures and the angiographic outcomes are shown. Abbreviations are defined in Tables $1 \& 4$.

$(0.95 \pm 0.48 \mathrm{~mm})$, follow-up \%DS $(68.1 \pm 14.8)$, and late luminal loss $(1.55 \pm 0.54 \mathrm{~mm})$ in the binary restenosis group were significantly different from those in the non binary restenosis group $(1.40 \pm 0.64,34.0 \pm 18.6,0.95 \pm 0.52 \mathrm{~mm}, 64.2 \pm 17.8$, $1.53 \pm 0.54 \mathrm{~mm}, 2.32 \pm 0.54 \mathrm{~mm}, 21.5 \pm 10.4$, and $0.17 \pm 0.49$ $\mathrm{mm}$, respectively) $(\mathrm{p}<0.001,<0.001,<0.05,<0.01,<0.01$, $<0.001$, and $<0.001$, respectively).

\section{Predictors of binary restenosis in 989 angiographic lesions}

The results of a logistic regression analysis performed to identify the predictors of binary restenosis after treatment with PES or SES are shown in Table 6. The multivariate analysis identified the following predictors as being significant: hemodialysis [odds ratio (OR), 4.34; 95\% CI: 1.91- 
Table 6. Predictors of Binary Restenosis

\begin{tabular}{cccc}
\hline & Odds ratio & $95 \% \mathrm{Cl}$ & $\mathrm{p}$ value \\
\hline Hemodialysis & 4.34 & $1.91-9.84$ & $<0.001$ \\
LCX ostium & 3.47 & $1.79-6.74$ & $<0.001$ \\
Side branch stenting & 2.86 & $1.59-5.15$ & $<0.001$ \\
RCA ostium & 2.64 & $1.02-6.79$ & $<0.05$ \\
\hline Length of stent & 1.02 & $0.99-1.05$ & 0.19 \\
LAD & 0.78 & $0.50-1.22$ & 0.28 \\
Acute gain & 1.27 & $0.72-2.25$ & 0.41 \\
Number of stents & 1.26 & $0.59-2.69$ & 0.55 \\
PES & 1.14 & $0.73-1.77$ & 0.57 \\
Pre-procedural \% DS & 1.01 & $0.98-1.03$ & 0.67 \\
CTO & 0.90 & $0.42-1.94$ & 0.80 \\
Pre-procedural MLD & 0.91 & $0.37-2.27$ & 0.84 \\
\hline
\end{tabular}

Predictors of binary restenosis analyzed by logistic regression analysis are shown. Abbreviations are defined in Tables 1 \& 4. The first 4 variables were significant predictors according to the multivariate analysis.

9.84; p<0.001), LCx ostium (OR, 3.47; 95\% CI, 1.79-6.74; $\mathrm{p}<0.001$ ), side-branch stenting (OR, 2.86; 95\% CI, 1.595.15 ; $\mathrm{p}<0.001$ ), and RCA ostium (OR, 2.64; 95\% CI, 1.02$6.79 ; \mathrm{p}<0.05)$. The length of stents, LAD, acute gain, number of stents, PES, pre-procedural \%DS, CTO, and preprocedural MLD were not significant predictors according to the multivariate analysis.

\section{Discussion}

This retrospective, nonrandomized, single-center study assessed the mid-term (mean follow-up interval of approximately 2.3 years) safety and efficacy of PES (TAXUS Express) for the treatment of complex lesions in Japan. The present study was undertaken because there have so far been only a few studies in Japan investigating the outcomes of using first generation DES by comparing the safety and efficacy of PES versus SES $(1,2)$. The present study was the first investigation of the impact of PES on complex lesions in patients with conventional predictors of SES failure. Our results demonstrated favorable midterm safety of the PES without definite ST for patients with various predictors commonly reported in Japan (binary restenosis, TLR, and severe cardiac events) $(3-5,10,11)$ (Table 1,2$)$ : more than $75 \%$ of the lesions were in patients with diabetes (12); approximately $20 \%$ of the lesions displayed calcification $(4,13,14),>10 \%$ were ostial $(4,15)$, diffuse lesions treated with long stents (mean length of stent more than 35 $\mathrm{mm})(15,16)$, and $>10 \%$ used the bifurcation 2-stent technique $(3,4,10,15)$. Among the present cohort, hemodialysis, a low EF, IVUS and age were the significant predictors of severe cardiac events (Table 3), which was consistent with the findings of previous reports $(3,5,10,17)$.

However, over a mean follow-up period of 2.3-2.4 years, the percentages of severe cardiac events after PES or SES placement (within a few percentages) were acceptable and more favorable compared to those in Western countries (18-20) (Table 1). Specifically, the rate of severe cardiac events in Western studies was approximately 7.5-14.5\% after one year, and not only were some of the predictors analyzed in the present study not included in those studies, but the percentages of patients with variables such as diabetes, calcification, ostial lesions, and use of the bifurcation 2stent technique were much higher in our study than in those studies (18-20). Accordingly, we herein provided the first demonstration of the mid-term safety of PES for the treatment of complex lesions with predictors of cardiac events, binary restenosis, and TLR in Japanese patients by evaluating 1,335 de novo native complex coronary stenosis lesions in a total of 840 patients.

The present nonrandomized study shows that PES and SES are statistically equivalent in terms of their efficacy with regard to the incidences of binary restenosis and TLR within 550 days (Table 4). Almost all of the variables were statistically equivalent between the groups at baseline in the angiographic follow up of all 989 lesions (Table 4), and the use of PES was not related to predictors of binary restenosis (Table 6). The mean late luminal loss after PES placement was 1.5-fold higher than that after SES placement. However, the overall binary restenosis ratio after PES placement for de novo native complex coronary lesions was statistically equivalent (a 12.8\% increase) to that after SES placement (Table 4). Therefore, despite less late luminal loss after SES placement, the use of SES was not more advantageous than the use of PES (e.g., it did not have a more potent antirestenotic effect by reducing the growth of intima), since the binary restenosis ratio was equivalent to that of PES. This finding is consistent with a previous report (21).

Therefore, our nonrandomized study demonstrates that the midterm safety and efficacy of PES and SES are statistically equivalent based on the 900-day clinical and 550-day angiographic outcomes among a selected complex cohort (Table 2, 4). Since PES increase the long-term patency (no increase in late loss from 6 months to 2 years after placement) (22) compared to SES (23), a long-term follow-up is needed to compare the angiographic efficacy between PES and SES. In addition, the present study showed the necessity of performing further investigations to examine the impact of the present second generation DESs on the failures of the first generation DESs (SES and PES) in order to raise the rate of complete revascularization.

The present study had several limitations that should be kept in mind when interpreting the results of the study. First, we utilized a retrospective, non-randomized, and single-center analysis. Since the duration of SES treatment was longer than that of PES, there was a large difference in the cohort numbers between the PES and SES groups. The present cohort had lower fewer patients on hemodialysis, predictors of cardiac events, and binary restenoses after the implantation than did a Japanese previous study (10). The lower rate of fu CAG among the PES group might also have 
underestimated the efficacy of PES (Table 2). Furthermore, the duration of dual antiplatelet therapy and the choice of thienopyridine agent (ticlopidine or clopidogrel) depended on the doctor's judgment. The number of patients who were noncompliant with or discontinued dual antiplatelet therapy due to surgical and bleeding complications was also not fully examined. However, there was no increase in the number of cardiac events or definite ST events (Table 1). Finally, other predictors of cardiac events, such as medications, renal dysfunction, and anemia were not examined.

\section{Conclusion}

This retrospective, nonrandomized study showed that, over a mean interval of 2.3-2.4 years, the midterm clinical and angiographic outcomes of PES placement for selected de novo native complex coronary lesions in Japanese patients were acceptable and statistically equivalent to those of SES placement.

The authors state that they have no Conflict of Interest (COI).

\section{References}

1. Fukumoto A, Otsuji S, Takiuchi S, et al. Comparison of realworld clinical outcomes between Cypher- and Taxus-eluting stents: the GARA-GARA study. Cardiovascular Interv Therap 25: 202-208, 2010.

2. Ishikawa T, Mutoh M, Nakano Y, et al. Post-discharge clinical and angiographic outcomes of patients presenting within 48 hours of STEMI treated with paclitaxel- or sirolimus-eluting stents. J Cardiol 2012(in press).

3. Kubota T, Ishikawa T, Mutoh M. Retrospective comparison of the clinical and angiographic outcomes of the sirolimus-eluting stent and the bare-metal stent in 2031 nonrandomized consecutive de novo native coronary lesions. Intern Med 50: 2463-2470, 2011.

4. Mutoh M, Ishikawa T, Hasuda T, et al. Predictors of target lesion revascularization and documented stent thrombosis beyond 30 days after sirolimus-eluting stent implantation-retrospective analysis in consecutive 1070 angiographic follow-up lesions. Circ J 71: 1328-1331, 2007.

5. Ikari Y, Kotani J, Kozuma K, Kyo E, Nakamura M, Yokoi H; JPMS Study Group. Assessment of sirolimus-eluting coronary stent implantation with aspirin plus low dose ticlopidine administration: one year results from CYPHER Stent Japan Post-Marketing Surveillance Registry (J-PMS). Circ J 73: 1038-1044, 2009.

6. Cheneau E, Satler LF, Escolar E, et al. Underexpansion of sirolimus-eluting stents: incidence and relationship to delivery pressure. Catheter Cardiovasc Interv 65: 222-226, 2005.

7. Kubota T, Ishikawa T, Nakano Y, et al. Retrospective comparison of clinical and angiographic outcomes after sirolimus-eluting and bare-metal stents implantation for nonrandomized consecutive 312 severe calcified lesions using rotablator. Int Heart J 52: 65-71, 2011.

8. Sakamoto H, Ishikawa T, Mutoh M, Imai K, Mochizuki S. Angi- ographic and clinical outcomes after sirolimus-eluting stent implantation to de novo ostial lesion of the right coronary artery: a retrospective study. Circ J 72: 880-885, 2008.

9. Cutlip DE, Windecker S, Mehran R, et al; Academic Research Consortium. Clinical end points in coronary stent trials: a case for standardized definitions. Circulation 115: 2344-2351, 2007.

10. Kimura T, Morimoto $T$, Nakagawa $Y$, et al. Antiplatelet therapy and stent thrombosis after sirolimus-eluting stent implantation. Circulation 119: 987-995, 2009.

11. Iakovou I, Schmidt T, Bonizzoni $\mathrm{E}$, et al. Incidence, predictors, and outcome of thrombosis after successful implantation of drugeluting stents. JAMA 293: 2126-2130, 2005.

12. Akin I, Bufe A, Schneider S, et al. Clinical outcomes in diabetic and non-diabetic patients with drug-eluting stents: results from the first phase of the prospective multicenter German DES. DE registry. Clin Res Cardiol 99: 393-400, 2010.

13. Budoff MJ, Shaw LJ, Liu ST, et al. Long-term prognosis associated with coronary calcification: observations from a registry of 25,253 patients. J Am Coll Cardiol 49: 1860-1870, 2007.

14. Kawaguchi R, Tsurugaya H, Hoshizaki H, Toyama T, Oshima S, Taniguchi K. Impact of lesion calcification on clinical and angiographic outcome after sirolimus-eluting stent implantation in realworld patients. Cardiovasc Revasc Med 9: 2-8, 2008.

15. Colombo A, Chieffo A. Drug-eluting stent update 2007: part III: technique and unapproved/unsettled indications (left main, bifurcations, chronic total occlusions, small vessels and long lesions, saphenous vein grafts, acute myocardial infarctions, and multivessel disease). Circulation 116: 1424-1432, 2007.

16. Shirai S, Kimura $T$, Nobuyoshi $M$, et al. Impact of multiple and long sirolimus-eluting stent implantation on 3-year clinical outcomes in the j-Cypher Registry. JACC Cardiovasc Interv 3: 180188, 2010.

17. Roy P, Steinberg DH, Sushinsky SJ, et al. The potential clinical utility of intravascular ultrasound guidance in patients undergoing percutaneous coronary intervention with drug-eluting stents. Eur Heart J 29: 1851-1857, 2008.

18. Morice MC, Colombo A, Meier B, et al. Sirolimus- vs paclitaxeleluting stents in de novo coronary artery lesions: the REALITY trial: a randomized controlled trial. JAMA 295: 895-904, 2006.

19. Simonton CA, Brodie B, Cheek B, et al. Comparative clinical outcomes of paclitaxel- and sirolimus-eluting stents: results from a large prospective multicenter registry--STENT Group. J Am Coll Cardiol 50: 1214-1222, 2007.

20. Mayor M, Malik AZ, Minor RJ Jr, et al. One-year outcomes from the TAXUS express stent versus cypher stent. Am J Cardiol 103: 930-936, 2009.

21. Agostoni P, Cosgrave J, Biondi-Zoccai G, et al. Angiographic analysis of pattern of late luminal loss in sirolimus- and paclitaxel-eluting stents. Am J Cardiol 99: 593-598, 2007.

22. Claessen BE, Beijk MA, Legrand V, et al. Two-year clinical, angiographic, and intravascular ultrasound follow-up of the XIENCE $\mathrm{V}$ everolimus-eluting stent in the treatment of patients with de novo native coronary artery lesions. The SPIRIT II Trial. Circ Cardiovasc Intervent 2: 339-347, 2009.

23. Kimura T, Morimoto $T$, Nakagawa $Y$, et al; on Behalf of the $j$ Cypher Registry Investigators. Very late stent thrombosis and late target lesion revascularization after sirolimus-eluting stent implantation: five-year outcome of the j-Cypher Registry. Circulation 125: 584-591, 2012.

(C) 2012 The Japanese Society of Internal Medicine http://www.naika.or.jp/imonline/index.html 